\title{
The role of HLA-typing in hematopoietic stem cells transplantation
}

\author{
Khomenko V. I. \\ Kyiv Center of Bone Marrow Transplantation, Kyiv, Ukraine \\ e-mail: vikhom@i.ua
}

\section{ABSTRACT}

The system of human leukocyte antigen (HLA) and HLA-typing were used to match a potential donor with a recipient for allogeneic hematopoietic stem cell transplantation (HSCT). The HLA matching between donor and recipient plays a key role in allogeneic HSCT. The mismatch of HLA can cause graft rejection, graft-versus-host disease and decrease of the survival in patients receiving grafts from both related and unrelated donors. The adverse HLA effect on the outcome depends on the total number of mismatched alleles/loci and the resolution level of the mismatch (antigen or allele level).

Thus, the final choice of compatible donor-recipient pairs should be based on high resolution molecular-genetic methods of HLA-typing. Serologic and molecular genetic methods of low resolution HLA-typing, which are cheaper than high-resolution HLA-typing, should be used for donor screening studies. HSCT from a fully compatible donor, matched high-resolution HLA-typing methods gives better results than from partially compatible. In some clinical circumstances, a partially compatible donor may be as effective as fully compatible. The selection of such a donor, taking into account the controversy of data from various literary sources, should be based on own research and experience. Creation and development of a Ukrainian database of donors with the HLA-haplotype specific to the indigenous population will make search of matching pairs of donor recipients more effective and cost-effective.

KEYWORDS: allogeneic hematopoietic stem cell transplantation (HSCT); human leucocyte antigens; HLA-typing

Allogeneic hematopoietic stem cell transplantation (HSCT) is an effective method for treating many malignant and non-malignant diseases of the hematopoietic system. To carry out such transplantation successfully, it is necessary to have a histocompatible family member or unrelated donor. Measures for the selection of histocompatible donorrecipient pairs for allogeneic transplantation include a careful analysis of human leukocyte antigens (HLA). This is necessary in order to minimize negative immunological effects, such as graft versus host disease (GVHD), graft rejection and other post-transplantation complications associated with increased mortality connected with transplantation and reduced overall survival [1-4].

One of the major factors contributing to the improvement of the allogeneic HSCT results was the progress made in the field of HLA studies. Substantial changes have occurred not only in the development of more advanced laboratory methods for rapid and accurate tissue typing, but also in a much deeper understanding of the HLA system, as well as the effects of HLA compatibility on the survival and development of complications associated with allogeneic HSCT. It is now considered that full HLA matching between the recipient and the donor, which is confirmed by high-resolution tissue HLA-typing methods, is associated with better clinical results $[4,5,11,18]$.

However, today in the world at both regional and global levels, there is a shortage of HLA-compatible donors, which is one of the main reasons that limits patients' access to allogeneic HSCT. The development of national and international donor registries of hematopoietic stem cells (HSC) has contributed to a significant increase in the number of allogeneic transplantations in the world using unrelated volunteer donors. Nevertheless, at the present stage of HSCT development, there is a significant gap between the demand and the supply of HLAcompatible donor material $[6-8,17]$, which leads to an increase in the use of haploidentical donors $[9,10]$. In this regard, we give an overview of research on current opportunities for providing allogeneic HSCT with histocompatible donor material, as well an attempt to address the problem of access to it using a partly HLA-compatible donor. This information will be useful for Ukraine, which does not have a significant experience in the development of allogeneic HSCT from related and unrelated donors.

Today in the world, HLA-typing is carried out by two methods serological and molecular genetic ones. The latter includes SSP (sequence 
specific primers), SSO (sequence specific oligonucleotides), and SBT (sequence-based typing) technologies. The introduction of high-resolution HLA-typing into clinical practice significantly improved survival after allogeneic HSCT by detecting «hidden» incompatibilities that could not be detected by the low-resolution serological or molecular genetic method. SSP technology enables you to perform both low- and high-resolution typing. The disadvantage of this technology is low performance. SSO technologies are relatively less labour intensive and higher productive. In this regard, it is more suitable for the formation of donor HSC registries, since, to create a register in the information base, data on the typing of tens of thousands of donors should be accumulated. SBT technology allows you to determine the complete nucleotide sequencing of DNA. Although it has the highest resolution, it is the most expensive. It is used in cases where there are problems with the interpretation of the results obtained from the use of technologies listed above [11].

The most important HLA determinants are six «classic» HLA polymorphic loci: A, B, C, DRB1, DQB1, and DPB1. Compatibility between donor and recipient in six loci (12/12) is rare. The compatibility in five HLA-A, $-B,-C,-D R B 1,-D Q B 1(10 / 10)$ loci in recent years is considered ideal. The current «gold standard» is a donor compatible in four alleles HLA-A, $-B,-C$, -DRB1 $(8 / 8)$. This statement is based on the analysis and synthesis of the results of many studies conducted by Shaw and c0-authors [12].

The best donor for allogeneic HSCT is the family histocompatible donor. Among family members, there is a high $(25 \%)$ probability that the patient and his sibling (brother or sister) will be completely histoidentical. With a probability of $50 \%$, you can find a haploidentical family donor. In addition, there is a $25 \%$ probability of a complete mismatch between the siblings [13].

With the increase in the number of children in the family, the probability of finding a histocompatible donor among several siblings increases. Given the average number of children in families in North America or Europe, less than $25-30 \%$ of patients requiring allogeneic transplantation have a chance to find histocompatible siblings [17]. Thus, the allogeneic HSCT in the remaining patients (about $70 \%$ ) depends on the successful search for a histocompatible unrelated donor in the national and international registries of the HSC donors and the umbilical cord blood banks. Despite the fact that in the world from 2002 to 2017 the number of volunteer donors rapidly increased from 8 million to more than 30 million, only about a half of the patients are able to find a histocompatible unrelated donor [14]

The great difficulty in identifying an ideal donor is related to the extremely high polymorphism of the HLA genes. In addition, most HSC donors are of European origin according to the World Bone Marrow Donor Worldwide (BMDW) registry. Studies conducted in Europe suggest that for non-European patients a non-specific donor with a degree of HLA compatibility of $9 / 10$ or $10 / 10$ can be identified for $60-80 \%$ patients and with a degree of compatibility of $12 / 12$ - for about $40 \%$ patients. The success of the search for patients of other racial and ethnic origin is much lower, especially for those of mixed origin [15].

The studies conducted in Germany at the University Hospital of Essen confirm this statement. The department of transfusion medicine in 2011 managed to complete the search for donors for $94 \%$ of German patients and $79 \%$ of migrants from Turkey within six weeks. They managed to find a suitable donor with a degree of HLA-compatibility $10 / 10$ for $70 \%$ German patients and for only $33 \%$ patients from Turkey [16].

Thus, the shortage of histocompatible non-related donors is one of the main causes limiting the availability of allogeneic HSCT at the global and regional levels. This is especially true of racial and ethnic minorities, as well illustrated by the study of Bergstrom and co-authors. Studies conducted in the United States have shown that the probability of finding a compatible donor and recipient among people of one racial origin is less than $1 / 10,000$. For example, this figure is $1 / 11000$ if the search is conducted among the white population. The same probability is set for Native Americans. However, the chance to find among white donor a compatible one for a Latino patient is 4 times lower than for white, for an
African American it is 10 times lower, and for Americans of Asian origin 20 times [17].

This situation has led to logical questions: «ls it possible to increase the availability of HSCT due to the use of a partly HLA-compatible donor?» and «lf so, which HLA non-compatibilities and in what clinical situations can be considered acceptable or unacceptable?». In order to answer the raised questions, we decided to analyze the current state of research in the field of HSCT, which are related to the above-mentioned aspect.

In the United States, Lee et al. conducted a retrospective analysis of 3857 allogeneic transplantations from non-native donors that were performed using myeloablative-conditioning regimens from 1988 to 2003. Donor-recipient pairs were examined by molecular-genetic typing of HLA-high resolution for HLA-A, -B, -C, -DRB1, -DQB1, -DQA1, -DPB1 and -DPA1 alleles. The compatibility of the patient and donor with HLA-A, -B, -C, and -DRB1 (8/8 compatibility) was associated with higher survival rates in patients. At least one or two HLA-A, -B, -C or -DRB1 incompatibilities (7/8 or $6 / 8$ ) would be associated with higher mortality. One-year survival in the first group of patients was $52 \%$, in the second $43 \%$, and in the third $-33 \%$. The authors conclude that patients tolerate the lack of compatibility of one HLA-B or HLA-C locus better than the HLA-A or HLA-DRB1 mismatch. Lack of compatibility of two or more loci threatens a significant risk of severe complications. HLA-DPB1 or -DQB1 loci mismatch was not associated with survival. However, if one or two HLA-A, -B, -C or -DRB1 loci were present, HLA-DQB1 locus incompatibility caused additional adverse effects on the results of HSCT. Therefore, when selecting donors, it should be preferred to be compatible for HLA-DQB1 if the candidates have the same degree of mismatch among other loci [18].

The degree of HLA compatibility between the donor and recipient plays an extremely important role in the HSCT, along with factors such as the diagnosis, stage of the disease, age of the patient, serum cytomegalovirus status, which can have a negative effect on the post-transplant period, and others [19].

Lee et al. studied combined effects on overall survival rate after HSCT of a number of factors: degree of donor compatibility, stage of disease, and age of patients. One-year survival in patients at an early stage of the disease after HSCT from unrelated donors with a degree of HLA compatibility of $8 / 8$, $7 / 8$ and $6 / 8$ was $63 \%, 52 \%$ and $39 \%$, and five-year-olds $-50 \%, 39 \%$ and $28 \%$, respectively. One-year survival of patients at the intermediate stage of the disease after unrelated transplantation from donors with a similar degree of HLA-compatibility was $48 \%, 40 \%$ and $32 \%$, and five-year one $32 \%, 27 \%$ and $22 \%$, respectively. At the late stage of the disease after HSCT from unrelated donors with a degree of HLA compatibility of $8 / 8$, $7 / 8$ and 6/8, one-year survival was $31 \%, 29 \%$ and $24 \%$, and five-year one $-17 \%, 15 \%$, and $10 \%$, respectively. Young patients under the age of 40 years at the early stages of the disease after an unrelated HSCT with a degree of compatibility of $8 / 8$ had the best survival rates. For this group, one-year survival rate was $67 \%$, and 5 -year one - $54 \%$ [18].

Crocchiolo et al. obtained similar data of the effect of a partially compatible donor on the results of HSCT in Italy. They conducted a retrospective analysis of the treatment results of 805 adult patients who received allogeneic HSCT from unrelated donors with oncohematologic diseases in Italy between 1999 and 2006. The recipient-donor pairs were examined by high-resolution HLA-typing of HLA-A, -B, -C, -DRB1, and -DQB1 alleles. The authors noted a significantly higher risk of overall mortality rate, non-recurrent mortality, transplant rejection, and acute graft-versus-host reaction (GVHR) in patients with HSCT from unrelated donor who had one or more HLA mismatches confirmed by low or high resolution tissue typing. The donor's mismatch in one HLA locus (compatibility $9 / 10$ ) at HSCT in acute leukemia patients in the first complete remission (early stage of the disease) increased the mortality risk compared to the fully compatible recipient donor pairs (10/10) who received transplantation at the same stage of the disease. However, in patients at a later stage of the disease, the above HLA mismatch did not affect mortality. These results confirm the benefit of a fully compatible donor (10/10) at an early stage of the disease. The authors assume that 
a partially compatible donor (9/10) may be selected for HSCT in patients at a late stage of the disease when it is not possible to quickly find a fully compatible donor (10/10) [20].

Morrisima et al. conducted a retrospective analysis of HSCT results from unrelated donors that were performed for oncohematologic diseases in Japan. The donors and patients, who had previously been typed by serological method for loci HLA-A, -B and -DRB1, underwent molecular genetic typing of HLA-A, -B, -C, -DRB1, and -DQB1 loci. 1298 donorrecipient pairs were examined. Researchers have been trying to identify the HLA alleles responsible for immunological effects, such as GVHR, graft rejection, and others that are associated with nonrecurring mortality and overall survival [21].

The patients were divided into two groups - standard and high risk. The first group included patients who underwent HSCT at the first complete remission, and the second one - patients who received transplantation at later stages of the disease.

The overall three-year survival after HSCT from fully compatible donors in the standard-leukaemia group was $65.4 \%$ and in high risk $43.1 \%$. In case of inconsistencies in one of the HLA-A or -B locus in the standard-risk group, the overall three-year survival rate was reduced to $39.9 \%$ and in the high-risk group to $23.9 \%$. The results of the three-year overall survival in standard and high-risk groups after transplantations from donors that had a mismatch in one of HLA-C and HLA-DR/DQ loci did not have statistically significant differences compared to HSCT from fully HLA-compatible donors [21]

A single mismatch in HLA-A, -B, -C, or -DRB1 allele was identified as an independent risk factor for the development of acute GVHD. Lack of HLA-C allele compatibility had a synergistic effect on the development of acute GVHD in combination with another allele mismatch. Lack of HLA-A and/or HLA-B allele's compatibility was a significant risk factor for chronic GVHD development. Incompatibility in allele HLA class I (A, B and/or C) caused significantly greater incidence of graft rejection compared with a fully compatible donor. There was no significant association between the incompatibility of the HLA-C allele and the development of leukaemia relapse. Lack of $\mathrm{HLA}-\mathrm{A}$ and/or -B allele compatibility significantly reduced overall survival in patients with leukaemia after HSCT of both standard and high risk. Mismatch in HLA class II (HLA-DRB1 and/or -DQB1) allele did not cause such an impact on overall survival. It was found that multiple HLA loci mismatches lead to a significant reduction in overall survival at leukaemia of both standard (39.1\%) and high-risk (15.9\%) groups [21].

Thus, a Japanese study has shown a significant role of HLA class I allele at unrelated HSCT. In particular, the impact of HLA-C alleles on the development of acute GVHD and survival was different from HLA-A or -B alleles. In Japanese patients, HLA-A and/or allele abnormalities significantly reduced survival, and there was no compatibility in HLA-C or -DRB1 and/or -DQB alleles [21]. Unlike the Japanese, the above research results of Lee et al., conducted in the United States, say that the lack of allele HLA-B or -C compatibility was better tolerated by American patients than HLA-A or -DRB1 ones [18].

In the United States, Flomenberg et al. investigated the effect of HLA compatibility between the donor and the recipient confirmed by methods of HLA-typing of various (low and/or high) resolution on the results of HSCT. To do this, 1874 pairs of donor recipients, who received HSCT from 1988 to 1996, were retrospectively screened using high-resolution HLA-typing. Typing was carried out in HLA-A, -B, -C, -DRB1, -DQA1, -DQB1, -DPA1 and -DPB1 alleles. Clinical effects related to differences in the degree of donor and recipient compatibility that arise in HLAtyping with high- and/or low-resolution methods, as well as their effect on transplantation results and the significance of these data for donor selection, were analyzed. The results show a strong impact of HLA-A, -B, - C or -DRB1 loci incompatibility on survival after unrelated HSCT. Lack of compatibility for one of these loci resulted in similar negative effects that were associated with a significant survival reduction, and the presence of several mismatches further worsened the result. Lack of HLA-A compatibility had a significant impact on the development of GVHD than in other loci described above. HLA-C incompatibility was strongly associated with transplant rejection. Lack of DQ and DP loci compatibility did not cause a significant adverse impact on the outcome [22].

The inconsistencies revealed by the low-resolution HLA-typing led to a more severe negative impact on survival after HSCT than the mismatches detected by high resolution typing. After all, the adverse effect on the results was more evident at transplantation from a donor selected by the method of low-resolution typing compared to those that were selected by methods of high-resolution typing. However, the latter were also associated with adverse results [22].

Thus, the analysis of the above [18, 20-22] and other world studies, generalized by Park, Shaw and co-authors $[4,12]$, suggests their controversy as to which HLA mismatch can be considered acceptable or tolerable in comparison with other one. There is a unanimity that the compatibility between donor and recipient in five loci HLA-A, -B, -C, -DRB1, -DQB1 (10/10) in recent years is considered ideal. Due to the insignificant effects of compatibility in HLA-DQB1 locus on the result of HSCT, a number of researchers consider the current "gold standard» for transplantation from an unrelated donor to be compatible in HLA-A, -B, $-\mathrm{C},-\mathrm{DRB} 1(8 / 8)$ alleles that are selected by high resolution HLA-typing. However, the lack of compatibility in DQB1 may result in additional negative effects if there is an incompatibility in another locus at the same time. Under certain circumstances, other mismatches may be acceptable depending on the clinical situation. One or more HLA-A, -B, -C, -DRB1 allele's mismatches are usually associated with worse survival. Clinical situations where one mismatch can be allowed include HSCT in patients who are at a late stage of the disease, when T-cell depletion and nonmyeloablative or reduced intensity conditioning regimens are used.

The effect of HLA-DPB1 is less clear due to the low probability of compatibility between the patient and the donor in this locus. The determination of the DPB1 HLA-locus can be done in some cases when there is more than one suitable donor to consider the degree and type of compatibility in the selection of donors. The question, which HLA mismatch and which locus should be preferred, remains open. The choice between HLA-A, -B, -C or DRB1 incompatible donor, taking into account the controversy of current research, should be based on local studies and experiences. 


\section{CONCLUSION}

Analysis of the published data demonstrates the feasibility of establishing and developing the national program of hematopoietic stem cells (HSC) donation in Ukraine. The system of human leukocyte antigens (HLA) and HLA typing were used to map the potential donor to the recipient for hematopoietic stem cell transplantation (HSCT). HLA-matching between donor and recipient plays a key role at allogeneic HSCT. The availability of suitable donors for patients who do not have genotypically HLA-compatible siblings has improved significantly due to the establishment of international registries of voluntary HLA-typed donors. However, for many patients, an ideal donor is not available. The mismatch of HLA genes may lead to transplant rejection, graft-versus-host reactions, and reduced survival rate of patients who received a graft from both related and non-related donors. The negative effect of HLA on the result depends on the total number of incompatible alleles/loci and the level of incompatibility resolution (antigen or allele level).

Thus, the final choice of the matching recipient-donor pair should be based on molecular-genetic methods of high resolution HLA-typing. Serologic and molecular genetic methods of low resolution HLA-typing, which are cheaper than high-resolution HLA-typing, should be used for donor screening studies. HSCT from a fully compatible donor, selected by high-resolution HLA-typing methods, yields better results than those that are partially compatible. The search algorithm should include studies of HLA-A, -B, -C, -DRB1, and -DQB1 loci, and in some cases -DPB1. In some clinical circumstances, a partially compatible donor may be as effective as a fully compatible one. The selection of such a donor, due to the controversy of data from various literary sources, should be based on own research and experience. Creation and development of Ukrainian database of donors with the HLA-haplotype inherent to the indigenous population will make finding a compatible donor-recipient pair more efficient and economically profitable.

\section{REFERENCES}

1. Nowak J. Role of HLA in hematopoietic SCT. Bone Marrow Transplantation. 2008; 42: 71-76.

2. Morichima Y, Kashiwase K, Matsuo K, et al. Biological significance of HLA locus matching in unrelated donor bone marrow transplantation. Blood. 2015; 125(7): 11891197.

3. Kekre N, Antin J. Hematopoietic stem cell transplantation donor sources in the 21st century: Choosing the ideal donor when a perfect match doesn't exist. Blood. 2014; 124(3): 334-343.

4. Park M, Seo JJ. Role of HLA in Hematopoietic Stem Cell Transplantation. Bone Marrow Research. 2012; doi: 10.1155/2012/680841.

5. Kuzmich EV, Alyansky AL, Ivanova NE. Analiz rezul'tatov allogennoy transplantatsii gemopoeticheskikh stvolovykh kletok v zavisimosti ot stepeni HLA-podbora patsienta i nerodstvennogo donora [Analysis of the results of allogeneic transplantation of hematopoietic stem cells depending on the degree of HLA selection of the patient and unrelated donor]. Onkogematologiya - Oncohematology. 2014; 9(3): 25-31. [In Russian]

6. Bubnova LN, Pavlova IE, Glazanova TV, et al. Registry donorov gemopoeticheskikh stvolovykh kletok [Donor Register of hematopoietic stem cell]. Biomeditsinskiy zhurnal Medline.ru - Biomedical Journal Medline.ru. 2015; 16: 751-758.

7. Makarenko OA, Alyanskiy AL, Ivaniva NE. Effektivnost' poiska nerodstvennogo donora gemopoeticheskikh stvolovykh kletok c pomoshch'yu rossiyskoy poiskovoy sistemy Bone Marrow Donor Search: opyt NII detskoy onkologii, gematologii i transplantologii im. R. M. Gorbachevoy [Effectiveness of Search for an Unrelated Donor of Hematopoietic Stem Cells using Russian Date Base «Bone Marrow Donor Search»: Experience of Raisa Gorbacheva Memorial Institute for Children Oncology, Hematology and Transplantation]. Klinicheskaya onkogematologiya - Clinical oncohematology. 2017; 10(1): 39-44. [In Russian]

8. Afanasiev BV, Zubarovskaya LS, Moiseev IS. Allogennaya transplantatsiya gemopoeticheskikh stvolovykh kletok u detey: nastoyashchee, problemy, perspektivy [Afanasiev BV, Zubarovskaya LS, Moiseev IS. Allogeneic hematopoietic stem cell transplantation in children: now, problems and prospects]. Rossiyskiy zhurnal detskoy gematologii i onkologii - Russian Journal of Children Hematology and Oncology. 2015; 2(2): 28-42. [In Russian]

9. Passweg JR, Baldomero $\mathrm{H}$, Bader $P$, et al. Use of haploidentical stem cell transplantation continues to increase: the 2015 European Society for Blood and Marrow Transplant activity survey report. Bone Marrow Transplantation. 2017; 52: 811-817.

10. Passweg JR, Baldomero H, Bader P, et al. Hematopoietic stem cell transplantation in Europe 2014: more than 40000 transplants annually. Bone Marrow Transplantation. 2016; 51: 786-792.

11. Loginova MA, Paramonov IV, Trofimova NP. Neodnoznachnosti pri provedenii HLA-tipirovaniya po tekhnologi SSO i popytka ikh razresheniya [Ambiguity in HLA-typing with SSO technology and attempting to resolve them]. Vestnik transplantologii i iskusstvennykh organov - Russian Journal of Transplantology and Artificial Organs. 2010; 12(4): 33-38. [In Russian]

12. Shaw $B E$, Arguello R, Garcia-Sepulveda CA, et al. The impact of HLA genotyping on survival following unrelated donor haematopoietic stem cell transplantation. British Journal of Haematology. 2010; 150: 251-258.

13. Balint B, Pavlovic M, Todorovic M. Recent strategies vs. challenges in stem cell-based grafting: from innovative research to clinical practice. Bilt Transfuziol. 2011; 57(1-2): 9-25.

14. Bone Marrow Donor Worldwide, Stem Cell Donor Registries Statistics. Available: http://www.bmdw.org/26-bmdw/news-and-publications/statistics/84-stem-celldonor-registries.

15. Tiercy JM. Unrelated Hematopoietic Stem Cell Donor Matching Probability and Search Algorithm. Bone Marrow Research. 2012; 2012. 8 p.

16. Elmaagacli AH. Unrelated transplantation: donors issues. Ulusal Kemik lilği Transplantasyonu ve Kök Hücre Tedavileri Kongresi. 08 - 10 Mart 2012, Antalya. Available: http://www.thd.org.tr/thdData/Books/232/unrelated-transplantation-donor-issues-ahmet-h-elmaagacli.pdf

17. Bergstrom T, Garratt R, Sheehan-Connor D. One Chance in a Million: Altruism and the Bone Marrow Registry. Departmental Working Papers, Department of Economics, UCSB, University of California, Santa Barbara, 2008. 41 p.

18. Lee SJ, Klein J, Haagenson M, et al. High-resolution donor-recipient HLA matching contributes to the success of unrelated donor marrow transplantation. Blood. 2007; 110: $4576-4583$

19. Gratwohl A. The EBMT risk score. Bone Marrow Transplantation. 2012; 47: 749-56. 
20. Crocchiolo R, Ciceri F, Fleischhauer $K$, et al. HLA matching affects clinical outcome of adult patients undergoing haematopoietic SCT from unrelated donors: a study from the Gruppo Italiano Trapianto di Midollo Osseo and Italian Bone Marrow Donor Registry. Bone Marrow Transplantation. $2009 ; 44: 571-577$.

21. Morishima $Y$, Sasazuki $T$, Inoko $H$, et al. The clinical significance of human leukocyte antigen (HLA) allele compatibility in patients receiving a marrow transplant from serologically HLA-A, HLA-B, and HLA-DR matched unrelated donors. Blood. 2002; 99: 4200-4206.

22. Flomenberg N, Baxter-Lowe LA, Confer D, et al. Impact of HLA class I and class II high-resolution matching on outcomes of unrelated donor bone marrow transplantation: HLA-C mismatching is associated with a strong adverse effect on transplantation outcome. Blood. 2004; 104: $1923-1930$

\begin{tabular}{|c|c|}
\hline 口祭回 & $\begin{array}{l}\text { ARTILLE ON THE SITE } \\
\text { TRANSPLANTOLOGY.ORG }\end{array}$ \\
\hline
\end{tabular}

The author indicates no potential conflicts of interest.

Received: July 12, 2017

Accepted: November 30, 2017 